\title{
Modelo simulador prático e de baixo custo para treinamento de timpanotomia com colocação de tubo de ventilação
}

\author{
Practical and inexpensive simulator model for tympanotomy training with grommet tube \\ placement
}

Modelo de simulador práctico y económico para entrenamiento de timpanotomía con colocación de tubo de ventilación

André dos Santos Brandão ${ }^{1 *}$, Jussandra Cardoso Rodrigues ${ }^{1}$, Felipe Xavier de Souza ${ }^{1}$, Jocyane de Souza Andrade', Ana Larisse Gondim Barbosa¹.

\begin{abstract}
RESUMO
Objetivo: Demonstrar um modelo de simulador cirúrgico, prático e de baixo custo, para a prática de timpanotomia com colocação de tubo de ventilação. Métodos: Desenvolvimento experimental de um modelo para treinamento cirúrgico, construído a partir de uma infra-estrutura com duas plataformas de madeira, uma como base e outra como suporte para o molde, interligadas por uma haste articulada de metal. O molde foi formado a partir de seringas serradas em um comprimento de $2,5 \mathrm{~cm}$, com um anel membranoso de Policloreto de Vinil fixado com um amarrilho de plástico em sua ponta, simulando respectivamente conduto auditivo externo e membrana timpânica. Resultado: O simulador após montado permite a prática do procedimento desejado, além de outros como aspiração, retirada de cerume e corpos estranhos do conduto adutivo externo, podendo ser executados com auxilio tanto do microscópio, como do endoscópio rígido. Conclusão: O modelo é uma alternativa barata e prática para treinamento de timpanotomia com colocação de tubo de ventilação e outros procedimentos da rotina otorrinolaringológica, e o treinamento com o uso do simulador permite ao usuário dominar as habilidades necessárias e se familiarizar com os equipamentos e instrumentais para a execução destes procedimentos no ambiente cirúrgico real.
\end{abstract}

Palavras-Chave: Otorrinolaringologia, Treinamento por simulação, Tecnologia de baixo custo.

\begin{abstract}
Objective: To demonstrate a practical and inexpensive surgical simulator model for the practice of tympanotomy with grommet tube placement. Methods: Experimental development of a model for surgical training, built from an infrastructure with two wooden platforms, one as a base and one as a support for the mold, interconnected by a hinged metal rod. The mold was formed from sawed syringes at a length of $2.5 \mathrm{~cm}$, with a vinyl polychloride membrane ring fixed with a plastic tie at its tip, simulating respectively external ear canal and tympanic membrane. Results: The simulator after mounting allows the practice of the desired procedure, as well as others such as aspiration, removal of cerumen and foreign bodies from the external ear canal, and can be performed with the aid of both the microscope and rigid endoscope. Conclusion: The model is a cheap and practical alternative to tympanotomy training with grommet tube placement and other routine otorhinolaryngological procedures, and training using the simulator allows the user to master the necessary skills and become familiar with the equipment and instruments to perform these procedures in the real surgical environment.
\end{abstract}

Key words: Otolaryngology, Simulation training, Low cost technology.

${ }^{1}$ Hospital Universitario Bettina Ferro de Souza, Belém-Pará. *E-mail: andrebrandao_2006@hotmail.com

SUBMETIDO EM: 1/2020

ACEITO EM: 1/2020

PUBLICADO EM: 2/2020

REAS/EJCH | Vol.Sup.n.41 | e2946 | DOI: https://doi.org/10.25248/reas.e2946.2020 Página 1 de 9 


\section{RESUMEN}

Objetivo: Demostrar un modelo de simulador quirúrgico práctico y económico para la práctica de timpanotomía con colocación de tubo de ventilación. Métodos: Desarrollo experimental de un modelo para entrenamiento quirúrgico, construido a partir de una infraestructura con dos plataformas de madera, una como base y la otra como soporte para el molde, interconectadas por una varilla metálica articulada. El molde se formó a partir de jeringas aserradas a una longitud de $2,5 \mathrm{~cm}$, con un anillo de membrana de policloruro de vinilo fijado con una atadura de plástico en su punta, simulando respectivamente el conducto auditivo externo y la membrana timpánica. Resultados: El simulador después del montaje permite la práctica del procedimiento deseado, así como otros, como la aspiración, la extracción de cerumen y cuerpos extraños del conducto auditivo externo, y se puede realizar con la ayuda del microscopio y el endoscopio rígido. Conclusión: El modelo es una alternativa económica y práctica al entrenamiento de timpanotomía con tubo de ventilación y otros procedimientos otorrinolaringológicos de rutina, y el entrenamiento con simulador permite al usuario dominar las habilidades necesarias y familiarizarse con los equipos e instrumentos para realizar estos procedimientos en el entorno quirúrgico real.

Palabras clave: Otolaringología, Entrenamiento simulado, Tecnología de bajo costo.

\section{INTRODUÇÃO}

A colocação de tubo de ventilação após timpanotomia para tratamento de otite média é um dos procedimentos cirúrgicos mais comumente executados pelos otorrinolaringologistas e um dos mais realizados em crianças (PARKER DM, et al., 2016). Havendo possibilidade de utiliza-lo como uma opção no tratamento das otites médias serosas e otites médias agudas de repetição (SEDERBERG-OLSEN JF, et al., 2019). Estas patologias podem acometer até $60 \%$ das crianças abaixo dos 2 anos de idade, com valores chegando a mais de $80 \%$ em crianças com malformações craniofaciais, podendo ambas estarem associadas com perda de audição e danos a orelha média (VANNESTE P e PAGE C, 2019). E apesar da alta frequência deste procedimento em nosso meio, existem pormenores que podem dificultar sua correta realização, e só através de uma curva de aprendizado muitas das vezes com históricos de falhas ou erros, pode-se alcançar a devido segurança em sua execução (WIET GJ, et al., 2011).

A colocação de tubo de ventilação é um procedimento considerado rápido, mas requer experiência prévia no manejo de microcirurgias, podendo ocorrer complicações durante sua execução (JIA W e AL-OMARI BA, 2018). Estas ocorrem em torno de $25 \%$ a $33 \%$ das cirurgias realizadas (NOBREGA M, 2002). Entre as complicações, uma das mais frequentes é a perfuração residual, sendo relatada em até $2 \%$ dos pacientes que receberam tubo de ventilação curta duração, e em $17 \%$ dos pacientes que receberam tubo de ventilação de longa duração, sendo a diferença entre estes o tempo médio de permanência posicionado através da membrana timpânica mantendo o pertuito da timpanotomia pérvio, sendo que o primeiro pode ficar em torno de 1 a 6 meses, e o segundo cerca de 12 meses, porém este podendo vir a ficar por mais tempo (PARKER DM, et al., 2016).

Entre as habilidades requeridas para a correta execução deste, como de outros procedimentos no conduto auditivo externo (microssucção de secreções, remoção de cerume e corpos estranhos), estão o correto manejo do microscópio ou endoscópio, habilidades manuais para manipulação de instrumentais em um pequeno espaço, associado a delicadeza no manejo do conduto auditivo e membrana timpânica, além da coordenação mão/olho durante a execução das tarefas desejadas (CLARK MPA, et al., 2016). Quando estes são realizados por médicos sem treinamento prévio, como ocorre com os residentes recém ingressados no programa de residência médica, acabam se tornando procedimentos prolongados, dolorosos e muitas das vezes não se obtendo o resultado desejado (MALEKZADEH S, et al., 2011).

Novas alternativas começaram a surgir no sentido de suplementar a "falta" de experiência, tão cobrada com o passar do tempo (WIET GJ, et al., 2017). A introdução de simuladores clínicos ou cirúrgicos no ensino da saúde, hoje em dia presentes em muitas das faculdades de medicina e hospitais mundo a fora, passaram 
a propiciar um ambiente seguro, livre de maiores riscos, para o treinamento das técnicas necessárias para a execução de situações clínicas ou procedimentos invasivos. Podendo-se repetir uma mesma situação, tática ou técnica por diversas vezes, em um cenário onde o erro torna-se apenas uma nova oportunidade de tentativa (THONE N, et al., 2016).

Os primeiros modelos relatados datam em torno da década de 70, e desde então novos projetos vieram surgindo, seja de pequenos projetos manuais, até mesmo simuladores mais elaborados e complexos, com alguns envolvendo realidade virtual (ARORA A, et al., 2014). Hoje em dia o treinamento através de simuladores já está bem estabelecido como parte vigente dos currículos das faculdades de medicina, permitindo a prática e aquisição de habilidades psicomotoras sem expor pacientes aos estágios iniciais da curva de aprendizados dos estudantes (DEUTSCH ES, et al., 2015). E nas especialidades cirúrgicas a simulação ganha maior destaque, sendo um ótimo meio, seja no ganho de novas habilidades, como na manutenção e aperfeiçoamento destas (DEUTSCH ES, et al., 2015).

Assim sendo, com o intuiuto de promover alternativa ao ganho de experiência dos residentes da área de otorrinolaringologia, principalmente aqueles recém ingressados a especialização, o objetivo deste trabalho é apresentar um projeto de construção de um simulador cirúrgico para a prática e aquisição de habilidades na execução da timpanotomia com colocação de tubo de ventilação.

\section{MÉTODOS}

Trata-se de uma pesquisa tecnológica para desenvolvimento experimental de um modelo de simulador cirúrgico para treinamento, realizado no Hospital Universitário Bettina Ferro de Souza, sem participação de outros locais. Pelas características do projeto, este dispensou a necessidade de aprovação de um Comitê de Ética em Pesquisa vigente, nem de qualquer outra instituição reguladora, devido ao não envolvimento de nenhum experimento e interferência direta ou indireta em qualquer tipo de ser vivo, além de prescindir confecção de termo formal de consentimento devido não envolver acesso a informações de pessoas físicas. Foi seguido certos princípios determinados como necessários para a criação do modelo/simulador para treinamento cirúrgico: efetividade, durabilidade, baixo custo, fácil montagem e acessibilidade para o médico residente.

O projeto para a criação do simulador seguiu os preceitos geralmente estipulados nas pesquisas de desenvolvimento tecnológico: idealização, análise de viabilidade do projeto e produção do protótipo. Desde o início até o término do produto, levaram em torno de 7 meses de desenvolvimento, havendo alterações ao longo da pesquisa, para melhor adaptação das tarefas exigidas, sendo que todo o custo da pesquisa ficou de responsabilidade do autor, não havendo financiamento de qualquer colaborador ou instituição financeira.

O simulador foi construído utilizando-se uma base de madeira com área e peso suficientes para obter um suporte adequado, permitindo estabilidade durante a execução das tarefas pretendidas. A base possui dimensões por volta de $20 \mathrm{~cm}$ de largura, cerca de $30 \mathrm{~cm}$ de comprimento e $5,5 \mathrm{~cm}$ de espessura. Outra prancha de madeira foi utilizada como apoio, onde pequenos furos foram realizados sobre esta, se tornando uma plataforma para posicionamento dos componentes que irão simular o conduto auditivo externo e membrana timpânica. A plataforma de apoio possui mesma largura e comprimento da base, porém com 1,5 $\mathrm{cm}$ de espessura.

Entre a base e o apoio, foi interposto uma haste de metal articulada, comumente utilizada como suporte de parede para monitores, que permite deslocações angulares do apoio enquanto a base permanece fixa, podendo vir a simular as diversas posições que a cabeça pode se encontrar durante a realização dos procedimentos.

Para mimetizar o conduto auditivo externo, foi utilizado como padrão seringas de $3 \mathrm{ml}$ e de $5 \mathrm{ml}$, onde estas foram marcadas e cortadas para apresentar um comprimento em torno de $2,5 \mathrm{~cm}$, semelhante ao órgão in vivo, com as marcações na seringa podendo ser niveladas no mesmo comprimento, permitindo um posicionamento mais retificado (reto) do anel membranoso ou com comprimentos diferentes, nos dando uma angulação da extremidade da seringa, simulando o próprio angulo da membrana timpânica. 
Para representar a membrana timpânica foi escolhido um anel membranoso de policloreto de vinila ou policloreto de vinil, também conhecido como PVC, um dos polímeros sintéticos de plástico mais produzido no mundo, com uma vasta possibilidade de aplicações, sendo comumente encontrado como vedação das tampas em coroa das garrafas de bebidas gaseificadas. Utilizando-se um pequeno amarrilho de plástico com cremalheira, foi fixado este anel membranoso na extremidade da seringa, com tensão suficiente para este ficar esticado, simulando uma resistência de quando manipulado a membrana timpânica. Além disto, é desenhado sobre o representante da membrana um pequeno ponto central e uma linha radial conectando $o$ ponto e a extremidade do círculo, representando respectivamente os umbus da membrana timpânica e a projeção do cabo do martelo sobre a membrana, que funcionarão como pontos de referência quando realizado qualquer procedimento sobre a membrana. Este sistema representado pela seringa e anel membranoso de PVC já fixado é então interposto em umas das cavidades feitas sobre a prancha de apoio, já feitos com dimensões distintas visando os diferentes modelos que podem ser construídos (Figura 1).

Figura 1 - Simulador montado, com supraestrutura simulando conduto auditivo externo e membrana timpânica.

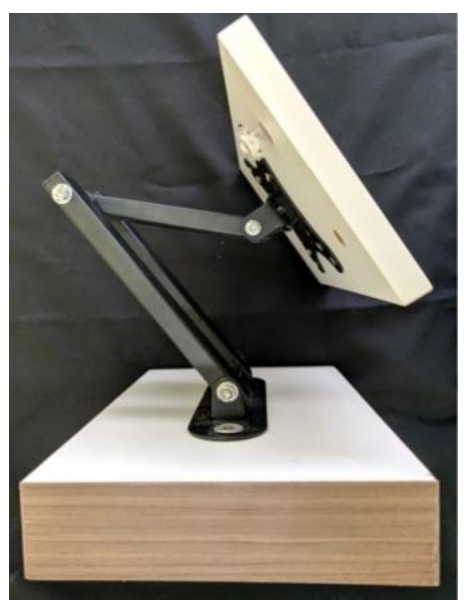

Fonte: Brandão AS, et al., 2020.

Este é então fixado, para melhor manipulação, utilizando o desenho anteriormente descrito como referência, podendo simular tanto a posição anatômica quanto cirúrgica da membrana, assim como outras possíveis posições (Figura 2).

Figura 2 - Anel membranoso de PVC com demarcações demilitando os quadrante da membrana timpânica, estando a figura $A$ representado a posição anatômica, com o paciente em pé ou sentado, e a figura $B$ representando a posição ciúrgica, com paciente deitado.

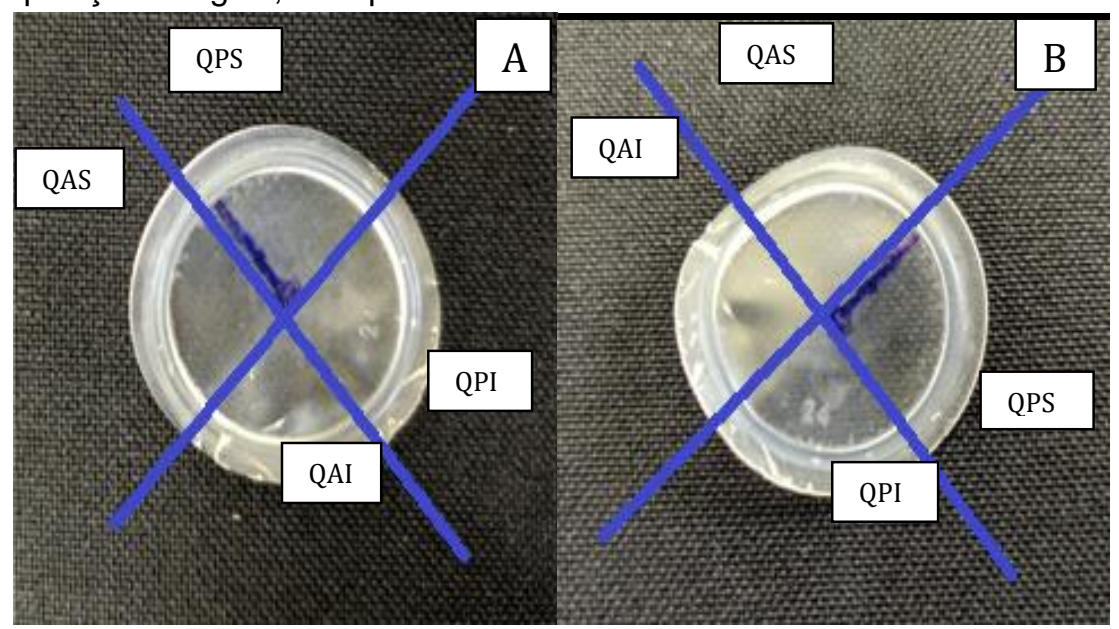

Legenda: QAS: quadrante antero-superior; QAI: quadrante antero-inferior; QPS: quadrante póstero-superior; QPI: quadrante postero-inferior. Fonte: Brandão AS, et al., 2020. 
Para poder simular outros procedimentos ambulatoriais, além do planejamento inicial da timpanotomia e colocação de tubo de ventilação, foi utilizado uma pluralidade de produtos: foi empregado creme de leite com pequena quantidade de corante alimentício para pode simular possíveis secreções, para treinamento da microaspiração de otorréias; para o cerume impactado e para a retirada de corpo estranho foi usado pequenos pedaços de esponja hemostática umedecidos colocados sobre a extensão do interior da seringa.

\section{RESULTADOS}

Após toda a infraestrutura montada, as simulações foram realizadas sobre microscopia direta com uso de microscópio cirúrgico da marca Weiss, com lente objetiva de 200x, padrão para as cirurgias otológicas, bem como sob visualização direta com endoscópio rígido de $2.7 \mathrm{~mm}$ e angulação de $30^{\circ}$ conectado a sistema integrado de vídeo Ferrari, modelo MFX 10G, com imagens projetadas em monitor de LCD da marca NDS. O procedimento para a colocação do tubo de ventilação é realizado inicialmente se fazendo um pequena incisão linear com um estilete micro cinzel ou lanceta em baioneta, preferencialmente em quadrante antero-inferior da membrana, neste caso representado pelo anel de PVC, e então com uma pinça hartmann (jacaré) para ouvido é posicionado o tubo de ventilação do tipo shepard sobre a incisão realizada e utilizando-se de um estilete de ponta reta em baioneta é realizado o encaixe do tubo de ventilação, para que este fique com metade de seu comprimento para dentro do interior da membrana timpânica e a outra metade para fora (Figura 3).

Figura 3 - Pequena incisão linear sobre quadrante antero-inferior da membrana, realizada com um estilete micro cinzel para ouvido (figura $A$ ), com tubo de ventilação já posicionado através da membrana com auxílio de estilete de ponta reta (figura B), por via endoscópica.

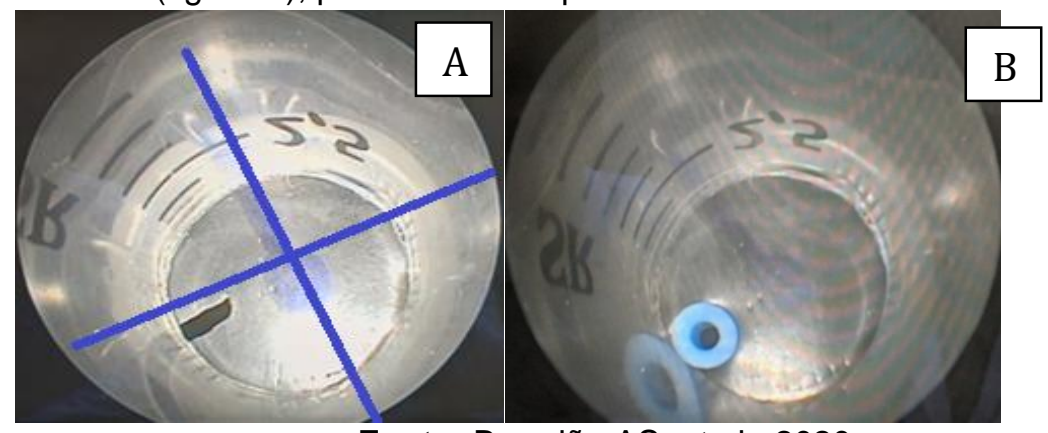

Fonte: Brandão AS, et al., 2020.

Outros procedimentos também foram testados, como a aspiração de secreções presentes no conduto auditivo externo. Foi realizado a mistura anteriormente descrita de creme de leite associado a corante alimentar da cor amarela, e colocado o conjunto permeando a extensão interna da seringa, então lançando mão de espéculos auriculares e ponta para aspiração de ouvido $n^{0} 15$, ponta está conectada a um aspirador ambulatorial. Em seguida é realizado aspiração de conteúdo com delicadeza a fim de simular a situação real, que em alguns casos traz bastante desconforto para o paciente (Figura 4).

Figura 4 - Presença de secreção sobre superfície interna do sistema sendo aspirada por ponteira conectada a aspirador ambulatorial, por microscopia.

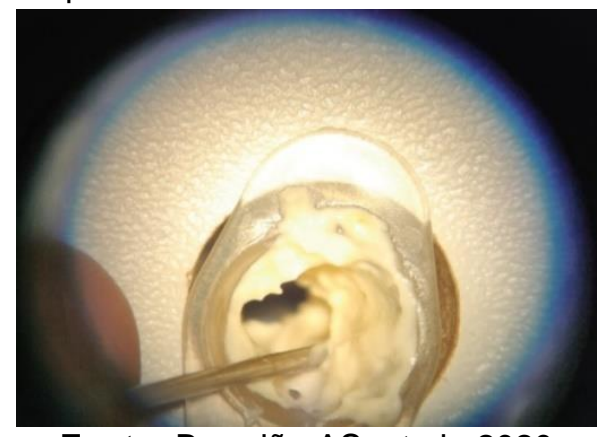

Fonte: Brandão AS, et al., 2020. 
Para a prática de retirada de cerume e retirada de corpos estranhos foi utilizado pequenos pedaços de esponja hemostática, sendo que estes tiveram que ser umidificados para gerar aderência entre sua superfície e o interior da seringa, e com uma cureta de cerume para ouvido foi realizado a retirada destes (Figura 5).

Figura 5 - Presença de esponja hemostática umedecida (seta preta) sobre superfície interna do sistema sendo retirada por cureta reta de cerume para ouvido, por microscopia.

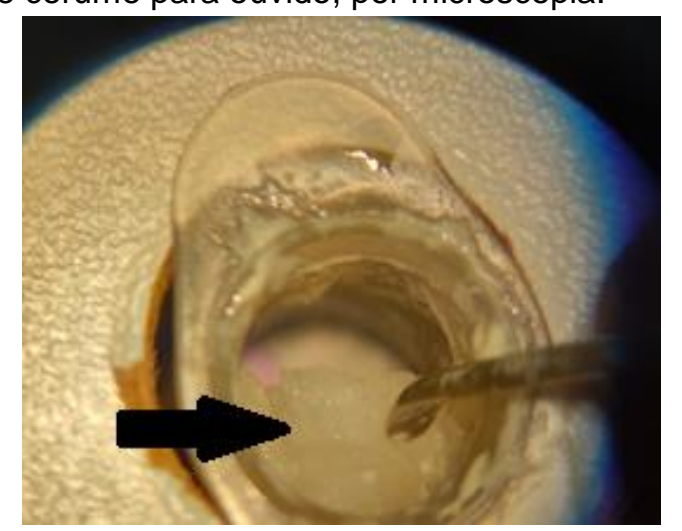

Fonte: BrandãO AS, et al., 2020.

\section{DISCUSSÃO}

A simulação se tornou parte integral da educação médica nos dias atuais, seja este desde o uso de manequins para treinamento de reanimação cardiopulmonar até simuladores de procedimentos e técnicas cirúrgicas (KURUP V, et al., 2017). O treinamento através das simulações comprovadamente possui efeitos positivos sobre o aprendizado e transferência de habilidades, alguns mesmo referindo efeitos positivos sobre o estado emocional durante a execução do procedimento, por habituação prévia dos prováveis resultados (MASIELLO I e MATTSSON A, 2017).

A realização dos procedimentos pela microscopia permite o residente adquirir familiaridade com 0 microscópio cirúrgico, sabendo melhor posicioná-lo, qual lente utilizar, qual magnificação escolher, além disso o ganho de destreza manual necessária na execução de procedimentos de microcirurgias, onde são utilizadas ambas as mãos para realizar movimentos discretos, fluídos e delicados em um pequeno campo cirúrgico, e permitindo também adequação em manipular os instrumentais usados em cirurgias otológicas (PREYER S, 2016).

A utilização da via endoscópica permite ao residente a entrar em contato a base de habilidades manuais necessários para as cirurgias por via endoscópica, pois estas diferem daquelas clássicas utilizadas nas microcirurgias. Nos procedimentos por via endoscópica é necessário que a mão não dominante do cirurgião esteja sempre segurando e posicionando o endoscópio durante a cirurgia, limitando este a utilização de apenas a mão dominante para a execução dos diversos procedimentos desejados, o que acaba sendo uma das grandes dificuldades no início da curva de aprendizado dos treinando (CLARK MPA, et al., 2019). Outro desafio é a necessidade de adequação a uma visualização do campo cirúrgico em duas dimensões, sem a apropriada noção de profundidade oferecida pela visão binocular do microscópio (DEDMON MM, et al., 2017).

Seguindo o preceito de baixo custo do modelo, toda a construção e montagem do projeto ficou em $\mathrm{R} \$$ 78,00 , e os componentes removíveis ficaram em torno de 40 centavos em média para cada nova tentativa, custos acessíveis para a classe pretendida entre os residentes na área de otorrinolaringologia. Apesar de alguns projetos na literatura buscarem a mesma acessibilidade de custo e peças para a fabricação do simulador, o preço foi divulgado em poucos destes, não podendo ser comparado o valor final do produto de alguns. Clark MPA, et al. (2016) refere gastos em torno de 100 dólares para o seu projeto, que convertendo à moeda brasileira vigente nos daria mais de 400 reais, preço bem acima do descrito neste trabalho, porém vale ressaltar que o valor convertido apenas não é suficiente para fazer a comparação de custos, sendo que este pode mudar baseado no poder de compra no mercado local desta moeda. 
A base e a plataforma de madeira utilizados para a confecção do projeto, juntamente com a haste articulada de metal interconectando estas através de parafusos, permitiram a robustez e durabilidade desejada ao produto final, escolha de produtos estas diferentes dos relatos na literatura (QUREISHI A e MOUALED D, 2014; ESTOMBA CMC, et al., 2015; CLARK MPA, et al., 2019). Jia W e Al-Omari BA (2018) optaram por materiais mais frágeis como vasilhas de papelão ou plástico para a base/suporte de simulador, porém com a vantagem de menor custo e maior acessibilidade, princípios também seguidos por Malekzadeh S, et al. (2011) e Qureishi A e Moualed M (2014). Clark MPA, et al. (2016) optaram por utilização de uma supraestrutura desenvolvida em impressora 3D, que apesar dos maiores custos, permitiram maior robustez e não necessidade de manutenção deste.

A utilização de seringas para a simulação do conduto auditivo externo já havia sido mencionada na literatura vigente. Estomba CMC, et al. (2015) e Malekzadeh S, et al. (2011) também utilizaram seringas de 3 $\mathrm{ml}$ para simular o diâmetro do conduto auditivo, mudando entre estes apenas a base do simulador e o material utilizado para a confecção do substituto da membrana timpânica. Ambos também se utilizaram de desenhos sobre a membrana para servir como ponto de referência para a execução dos procedimentos. Jia W e AlOmari BA (2018) utilizaram tubos endotraqueais para o simulador do conduto, sendo a vantagem de este ser a possibilidade de simular as tortuosidades do conduto auditivo externo, porém maior custo e menor acessibilidade a este. Qureishi A e Moualed D (2014) utilizaram pequenos containers de swabs para coleta de material para microbiologia, enquanto Jesudason WV e Smith I (2004) utilizaram capas descartáveis para um tipo de termômetro auricular. Já Clark MPA, et al. (2016) utilizaram um constructo próprio, mais complexo, desenvolvido em impressora 3D na forma de novelo, acoplado a outro constructo mimetizando a orelha média. Apesar dos diferentes designs, todos cumprem o mesmo papel de suporte para a colocação do algo que irá simular a membrana timpânica.

Não há outros trabalhos que descrevam a utilização dos anéis membranoso de PVC como substituto à membrana timpânica, nem mesmo o uso de amarrilhos para a fixação deste, porém este conjunto permitiu uma boa tensão a manipulação da membrana, simular a encontrada no procedimento in vivo. Há a descrição de uso de filmes de PVC na moldagem para simular a membrana, porém este de consistência mais frágil e maleável do que os anéis membranosos do mesmo material em questão (BAER S, et al., 1990). Outros materiais são descritos para a simulação da membrana timpânica, desde luvas plásticas utilizadas por Qureishi A e Moualed D (2014), membranas de látex por Estomba CMC et al. (2015), papel celofane por Duijvestein M e Borgstein J (2006) e papéis de cigarro/seda utilizados por Clark MPA et al. (2016), entre outros. Não há métodos de comparação para qual material entre os utilizados apresentaria uma melhor semelhança a peça anatômica, até mesmo por que estes estão conectados a infraestruturas diferentes e sobre tensões diferentes, o que pode mudar o comportamento do material em si, porém todos parecem cumprir o papel de forma satisfatória, permitindo execução da timpanotomia e colocação do tubo de ventilação.

O simulador desenvolvido neste trabalho permite a angulação da plataforma de apoio e alteração em sua altura, podendo a vir se adequar a diferentes situações seja na simulação de uma tarefa específica ou mesmo sobre a disponibilidade sobre onde apoiar o simulador. Grande parte dos simuladores descritos prezaram por confeccionar seus projetos para se ter uma inclinação durante a manipulação do sistema, dentre estes Jia W e Al-Omari BA (2018), Clark MPA, et al. (2016) e Estomba CMC, et al. (2015), para poder situar uma similaridade com as posição da cabeça durante a realização do procedimento, porém nenhum destes permitiram a possibilidade de alterar a angulação destes, o que acabou se provando como uma característica de adaptabilidade prática e funcional do presente projeto.

Durante realização das tarefas foi notado algumas limitações do projeto inicial, onde não foi possível a simulação de aspiração do glue-ear ou secreções através da incisão realizada na membrana, passo importante na timpanotomia com colocação de tubo de ventilação, impasse também encontrado por outros autores durante a confecção de seus projetos (JIA W e AL-OMARI BA, 2018; CLARK MPA, et al., 2016; ESTOMBA CMC, et al., 2015; QUREISHI A e MOUALED D, 2014; DUIJVESTEIN M e BORGSTEIN J, 2006). Leong A, et al. (2006) superaram este obstáculo, reproduzindo este feito através de um sistema construído a partir de um recipiente de lidocaína spray, um pequeno ducto de silicone cortado e um pedaço de luva de 
borracha, preenchendo este primeiro com solução de amido de milho, para simular a viscosidade apropriada do glue-ear, permitindo que esta solução pudesse ser aspirada após realização de incisão sobre a membrana.

A possibilidade de treinamento de outros procedimentos no projeto, como a aspiração de secreções presente em conduto, retirada de cerume ou corpos estranhos também foram pensadas por outros autores. Ismail-Koch H, et al. (2008) descreve a colocação de água com espessantes alimentares para simulação da aspiração de diferentes consistências de debris, baseado na proporção de água e espessante usado. Qureishi A e Moualed D (2014) utilizaram uma solução corada de azul, não identificando qual substância, para simular aspirações de possíveis secreções. Clark MPA, et al. (2016) utilizaram líquidos com espessante alimentar para as secreções, e massa de modelar para cerume e para corpos estranhos.

Apesar de ainda passível de alterações, o projeto demonstrou potencial para auxiliar residentes na área de otorrinolaringologia a ganhar e aprimorar as habilidades manuais destes, permitindo uma maior familiaridade com os procedimentos tão executados na prática desta especialidade. Além disso, o treinamento prévio com o uso do simulador permitiria dominar as habilidades necessárias e se familiarizar com os equipamentos e instrumentais para a execução do procedimento de timpanotomia com colocação de tubo de ventilação quando este fosse realizado em pacientes, melhorando a desempenho cirúrgico e diminuindo possíveis riscos de complicações no intraoperatório (CLARK MPA, et al., 2016).

\section{CONSIDERAÇÕES FINAIS}

O simulador após montado cumpriu seu papel estipulado no início do projeto, conseguindo manter os custos limitados, boa durabilidade, com necessidade de pouca manutenção e praticidade em conseguir e substituir os materiais necessários para novas tentativas. Todos os procedimentos intencionados foram possíveis de serem realizados, tanto por via microscópica quanto por endoscópica, porém alguns com limitações. Melhorias ainda são necessárias no projeto para maior adequação deste às práticas propostas, podendo influenciar mais ainda sobre a aquisição das habilidades manuais imprescindíveis na rotina otorrinolaringológica. Após melhorias do simulador e com a validação deste, futuros estudos esperam por demonstrar o real grau de utilidade do modelo em ajudar médicos residentes na execução dos procedimentos pretendidos, podendo assim quantificar o ganho de experiência provida por este.

\section{REFERÊNCIAS}

1. ANDERSEN SAW. Virtual reality simulation training of mastoidectomy - studies on novice performance. Danish Medical Journal, 2016; 63(8).

2. ARORA A, et al. Virtual reality simulation training in Otolaryngology. International Journal of Surgery, 2014; 12: 8794.

3. CLARK MPA, et al. An endoscopic Ear Trainer for the low-resource setting. The Journal of Laryngology \& Otology, 2019; 133(2).

4. CLARK MPA, et al. Development and validation of a low-cost microsurgery Ear Trainer for low-resource settings. The Journal of Laryngology \& Otology, 2016; 130: 954-961.

5. COSTA FG. Manutenção das estruturas metálicas com utilização dos ensaios não destrutivos. COSTRUMETAL, $2012 ; 2$.

6. DEDMON MM, et al. Development and Validation of a Modular Endoscopic Ear Surgery Skills Trainer. Otology \& Neurotology, 2017; 38.

7. DEUTSCH ES, et al. Simulation Activity in Otolaryngology Residencies. Otolaryngology-DICKSON, G. Acute Otitis Media. Primary Care: Clinics in Office Practice, 2014; 41:11-18.

8. DUIJVESTEIN M, BORGSTEI J. The Bradford grommet trainer. Clinical Otolaryngology, 2006; 31: 163-166.

9. ESTOMBA CMC, et al. The Vigo grommet trainer. European Annals of Otorhinolaryngology, Head and Neck diseases, 2015; 132: 53-55.

10. ISMAIL-KOCH H, et al. The ear trainer model. Clinical Otolaryngology, 2008; 33: 629-640.

11. JESUDASON WV, SMITH I. How we do it: the Bradford grommet trainer: a model for training in myringotomy and grommet insertion. Clinical Otolaryngology, 2005; 30: 364-383.

12. JIA W, AL-OMARI B. A versatile grommet trainer. THE CLINICAL TEACHER, 2018; 15: 1-3.

13. KURUP V, et al. Role of in-situ simulation for training in healthcare: opportunities and challenges. Current Opinion in Anesthesiology, 2017; 30.

14. LEONG A, et al. Artificial Ear: A Training Tool for Grommet Insertion and Manual Dexterity. Karger, 2006; 68: 115117. 
15. MALEKZADEH S, et al. A Model for Training and Evaluation of Myringotomy and Tube Placement Skills. The Laryngoscope, 2011; 121: 1410-1415.

16. MARTIN-PEREZ B, et al. Advances in Surgery Channel: the future of surgical education. Journal of Surgical Simulation, 2017; 4: 5-9.

17. NOBREGA M. Tubos de ventilação. Jornal de Pediatria, 2002; 78(6): 446-448.

18. OKADA DM, et al. Simulador cirúrgico para treinamento de dissecção do osso temporal. Brazilian Journal of Otorhinolaryngology, 2010; 76(5): 575-578.

19. PREYER S. Endoscopic ear surgery - a complement to microscopic ear surgery. HNO, 2017; 65(1): $29-34$.

20. QUREISHI A, MOUALED D. The Northampton microsuction and grommet trainer. Clinical Otolaryngology, $2014 ; 39$ : 400-401.

21. SEDERBERG-OLSEN JF, et al. Grommets in otitis media: a 25-year follow up. Acta Oto-Laryngologica, 2019.

22. SGAI RD. Fatores que afetam o tratamento para preservação de madeiras. Campinas, 2000. Dissertação (Mestrado em Engenharia Civil) - Faculdade de Engenharia Civil da Universidade Estadual de Campinas, Campinas, 2000.

23. THONE N, et al. Simulación en Otorrinolaringología: una herramienta de ensenanza y entrenamiento. Acta Otorrinolaringologica Española - Journal, 2016; 67: 115-120.

24. VANNESTE P, PAGE C. Otitis media with effusion in children: Pathophysiology, diagnosis, and treatment. A review. Journal of Otology, 2019; 14: 33-39.

25. WIET GJ, et al. Otologic Skills Training. Otolaryngologic Clinics of North America - Journal, 2017; 50: 933-945.

26. WIET GJ, et al. Training and Simulation in Otolaryngology. Otolaryngologic Clinics of North America - Journal, 2011; 44: 1333-1350. 